\title{
Ventilação Mecânica na Crise de Asma Aguda
}

\author{
Mechanical Ventilation in Acute Asthma Crisis
}

\author{
Carmen Sílvia Valente Barbas ${ }^{1}$, Bruno do Valle Pinheiro ${ }^{2}$, Arthur Vianna ${ }^{3}$, \\ Ricardo Magaldi ${ }^{4}$, Ana Casati ${ }^{5}$, Anderson José ${ }^{6}$, Valdelis Novis Okamoto ${ }^{7}$
}

\section{RESUMO}

JUSTIFICATIVA E OBJETIVOS: Em 2000, foi publicado o II Consenso Brasileiro de Ventilação Mecânica. Desde então, o conhecimento na área da ventilação mecânica avançou rapidamente, com a publicação de inúmeros estudos clínicos que acrescentaram informações importantes para o manuseio de pacientes críticos em ventilação artificial. Além disso, a expansão do conceito de Medicina Baseada em Evidências (MBE) determinou a hierarquização das recomendações clínicas, segundo o rigor metodológico dos estudos que as embasaram. Essa abordagem explícita vem ampliando a compreensão e a aplicação das recomendações clínicas. Por esses motivos, a AMIB - Associação de Medicina Intensiva Brasileira - e a SBPT - Sociedade Brasileira de Pneumologia e Tisiologia - julgaram conveniente a atualização das recomendações descritas no Consenso anterior. Dentre os tópicos selecionados a Ventilação Mecânica na Crise de Asma foi um dos temas propostos. O objetivo deste estudo foi descrever os pontos mais importantes relacionados à ventilação mecânica durante a crise de asma e sugerir as principais abordagens terapêuticas.

MÉTODO: Objetivou-se chegar a um documento suficientemente sintético, que refletisse a melhor evidência disponível na literatura. A revisão bibliográfica baseou-se na busca de estudos através de palavraschave e em sua gradação conforme níveis de evidência. As palavras-chave utilizadas para a busca foram: Ventilação mecânica na asma: asthma and mechanical ventilation.

1. Hospital das Clínicas da Faculdade de Medicina da Universidade de São Paulo (SP)

2. Universidade Federal de Juiz de Fora (MG)

3. UTI da Clínica São Vicente (RJ)

4. Hospital Israelita Albert Einstein (SP)

5. Universidade Federal do Espírito Santo (ES)

6. UTI da Santa Casa de São Paulo (SP)

7. UTI do Hospital AC Camargo (SP)

(C)Associação de Medicina Intensiva Brasileira, 2007
RESULTADOS: São apresentadas recomendações quanto aos modos ventilatórios e aos parâmetros a serem aplicados quando do ajuste do ventilador, além da monitoração recomendada. Apresentam-se ainda, técnicas alternativas que possam ser utilizadas.

CONCLUSÕES: Estratégias protetoras de ventilação mecânica são recomendadas durante a ventilação mecânica de paciente asmático grave.

Unitermos: Consenso, crise de asma, recomendação, ventilação mecânica

\section{SUMMARY}

BACKGROUND AND OBJECTIVES: The II Brazilian Consensus Conference on Mechanical Ventilation was published in 2000. Knowledge on the field of mechanical ventilation evolved rapidly since then, with the publication of numerous clinical studies with potential impact on the ventilatory management of critically ill patients. Moreover, the evolving concept of evidence - based medicine determined the grading of clinical recommendations according to the methodological value of the studies on which they are based. This explicit approach has broadened the understanding and adoption of clinical recommendations. For these reasons, AMIB - Associação de Medicina Intensiva Brasileira and SBPT - Sociedade Brasileira de Pneumologia e Tisiologia - decided to update the recommendations of the II Brazilian Consensus. Mechanical ventilation in the asthma attack has been one of the updated topics. Describe the most important topics on the mechanical ventilation during the asthma attack and suggest the main therapeutic approaches.

METHODS: Systematic review of the published literature and gradation of the studies in levels of evidence, using the key words "mechanical ventilation" and "asthma".

RESULTS: We present recommendations on the ventilatory modes and settings to be adopted when ventilating a patient during an asthma attack, as well as the recommended monitoring. Alternative ventilation techniques are also presented. 
CONCLUSIONS: Protective ventilatory strategies are recommended when ventilating a patient during a severe asthma attack.

Key Words: asthma, consensus, mechanical ventilation, recommendation.

\section{INTRODUÇÃO}

A crise de asma aguda é responsável por cerca de $2 \%$ das admissões em unidades de terapia intensiva (UTI). Cerca de metade desses pacientes necessitam de suporte ventilatório mecânico invasivo nas primeiras 24 horas, com mortalidade hospitalar de cerca de 10\%. Trata-se de pacientes jovens (média de aproximadamente 40 anos) e com maior prevalência do sexo feminino. Os fatores associados ao uso da ventilação mecânica (VM), corrigidos para o escore de gravidade APACHE II, são parada cardiorrespiratória antes da admissão hospitalar, lesão neurológica, hipoxemia e hipercapnia. O principal fator associado à mortalidade hospitalar da crise asmática é a parada cardiorrespiratória antes da admissão hospitalar e esforços devem ser feitos no sentido de prevenção desses episódios ${ }^{1,2}$.

É importante salientar que, ao contrário de outras áreas da ventilação mecânica, as recomendações para manuseio da crise asmática baseiam-se em número modesto de estudos fisiológicos, com amostras relativamente pequenas de pacientes. Essas recomendações são baseadas na prevenção de iatrogenias, como o barotrauma, que estavam associadas a complicações graves e ao óbito. A seguir serão apresentadas aplicações de estratégias protetoras de ventilação e a hipercapnia permissiva reduziram a taxa de mortalidade desses pacientes.

\section{INDICAÇÕES DA VENTILAÇÃO MECÂNICA INVASIVA}

As principais indicações para intubação traqueal e ventilação mecânica na crise de asma aguda são ${ }^{3-5}$ :

- Parada respiratória ou cardiorrespiratória;

- Esforço respiratório progressivo e sinais de fadiga;

- Alteração grave do estado de consciência (agitação ou sonolência);

- Retenção progressiva de gás carbônico;

- Hipoxemia não corrigida pela suplementação de oxigênio com máscara $\left(\mathrm{PaO}_{2}<60 \mathrm{mmHg}\right.$ ou $\mathrm{SaO}_{2}$ $<90 \%)$.

\section{OBJETIVOS DA VENTILAÇÃO MECÂNICA}

São os objetivos do suporte ventilatório na crise asmática aguda:

- Diminuir o trabalho respiratório imposto pelo aumento de resistência das vias aéreas e pelos níveis crescentes de hiperinsuflação durante a crise grave ${ }^{3,6}$;

- Evitar barotrauma, mesmo que para isso seja necessária a utilização da hipoventilação controlada ou hipercapnia permissiva. Essa estratégia tem reduzido a mortalidade associada à ventilação mecânica na crise de asma aguda, em séries de casos ventilados com hipercapnia permissiva, comparados à ventilação convencional ${ }^{3,7}$;

- Manter a estabilidade do paciente, enquanto o tratamento medicamentoso, com broncodilatadores e corticosteróides, reduz a resistência das vias aéreas, revertendo a crise de asma e permitindo que o paciente reassuma a respiração espontânea.

O suporte ventilatório na crise asmática pode ser invasivo (intubação traqueal) ou não-invasivo. O suporte ventilatório não-invasivo na crise asmática é detalhado no presente Consenso no capítulo referente à Ventilação Não-Invasiva.

\section{MODO VENTILATÓRIO}

Recomendação: Não há dados que determinem superioridade da ventilação com pressão controlada sobre a ventilação com volume controlado, ou vice-versa. No entanto, a ventilação com pressão controlada, com monitorização do volume-corrente expirado, oferece mais segurança para os pacientes em crise de asma aguda, diminuindo os riscos de ocorrência de auto-PEEP e barotrauma.

\section{Grau de Recomendação: D (Quadro 1)}

Comentário: Embora o controle da hiperinsuflação possa ser alcançado e controlado mais facilmente com o modo pressão-controlada, ele também pode ser obtido com ajustes adequados e monitoração da ventilação em modo volume-controlado. Por outro lado, na pressão-controlada, a piora na mecânica pulmonar pode comprometer a ventilação, assim como a melhora (tratamento do quadro obstrutivo) pode levar a aumentos indesejáveis do volume-corrente, exigindo, portanto, o mesmo nível de monitoração para detecção e correção dessas eventuais alterações. É fundamental que a equipe responsável esteja completamente familiarizada com o modo ventilatório a ser empregado ${ }^{8-11}$. 
Quadro 1 - Graus de Recomendação

\begin{tabular}{ll}
\hline Graus de Recomendação & Tratamento/Prevenção \\
\hline A & Revisão Sistemática (com homogeneidade) de ensaios clínicos controlados e aleatórios \\
& Ensaio clínico controlado e aleatório com intervalo de confiança estreito \\
& Resultados terapêuticos do tipo "tudo ou nada" \\
\hline B & Revisão sistemática (com homogeneidade) de estudos de coorte \\
& Estudo de coorte (incluindo ensaio clínico aleatório de menor qualidade) \\
& Observação de resultados terapêuticos (outcomes research) / estudo ecológico \\
& Revisão sistemática (com homogeneidade) de estudos caso-controle \\
& Estudo caso-controle \\
\hline C & Relato de casos (incluindo coorte ou caso-controle de menor qualidade) \\
\hline D & Opinião de especialista sem avaliação crítica ou baseada em matérias básicas (estudo fisiológico ou es- \\
& tudo com animais) \\
\hline
\end{tabular}

\section{AJUSTE DOS PARÂMETROS VENTILATÓRIOS}

Recomendação: Os parâmetros ventilatórios devem ser ajustados para diminuir a hiperinsuflação pulmonar, o que pode ser conseguido com a redução do volumeminuto e o prolongamento do tempo expiratório.

Grau De Recomendação: C

Comentário: A crise de asma caracteriza-se por grande aumento da resistência das vias aéreas, decorrente de broncoespasmo, inflamação nas paredes e acúmulo de secreção na luz brônquica. A hiperinsuflação que se instala tem importante papel na fisiopatologia da crise de asma e constitui um dos principais pontos a serem considerados na ventilação mecânica. A hiperinsuflação reduz o retorno venoso e pode, por compressão dos capilares pulmonares, aumentar a resistência na circulação pulmonar. Com isso, pode haver diminuição do débito cardíaco e hipotensão arterial. Além disso, como o aumento de resistência nas vias aéreas não é homogêneo, áreas menos envolvidas podem receber grandes volumes de ar (gerando altas pressões transalveolares) durante a ventilação corrente e podem romper-se, gerando barotrauma. A hiperinsuflação também impõe maior trabalho respiratório ao paciente, incluindo maior trabalho elástico, contração muscular inspiratória para vencer a auto-PEEP antes de se ter movimentação de ar para os pulmões e contração da musculatura expiratória para auxiliar a exalação. Por fim, a hiperinsuflação altera a curvatura do diafragma, conferindo-Ihe menor eficiência. Todos esses efeitos deletérios da hiperinsuflação justificam o ajuste dos parâmetros da ventilação mecânica com o objetivo de diminuí-la ${ }^{12,13}$.

Os principais parâmetros a serem ajustados incluem:

\section{VOLUME-CORRENTE}

Recomendação: Utilizar volumes-correntes baixos, de 5 a $7 \mathrm{~mL} / \mathrm{kg}$

\section{Grau De Recomendação: B}

Comentário: Estudos de observação de resultados terapêuticos concluíram que a utilização de volumes-correntes baixos (5 a $7 \mathrm{~mL} / \mathrm{kg}$ ) em crises de asma aguda, com conseqüente hipercapnia permissiva, diminuiu a mortalidade, se comparados com pacientes que foram ventilados de maneira convencional (volume-corrente acima de $10 \mathrm{~mL} / \mathrm{kg})^{7}$.

\section{PRESSÕES INSPIRATÓRIAS}

Recomendação: Deve-se manter a pressão de pico inspiratório abaixo de $50 \mathrm{cmH}_{2} \mathrm{O}$ e a pressão de platô abaixo de $35 \mathrm{cmH}_{2} \mathrm{O}$ ou a medida de auto-PE$\mathrm{EP}<15 \mathrm{cmH}_{2} \mathrm{O}$ para se evitar a hiperinsuflação e ocorrência de barotrauma com suas conseqüências.

\section{Grau de Recomendação: B}

Comentário: Estudos de observação de resultados terapêuticos concluíram que níveis de pressão de platô acima de $35 \mathrm{cmH}_{2} \mathrm{O}$ e de auto-PEEP acima de $15 \mathrm{cmH}_{2} \mathrm{O}$ estavam relacionadas à maior incidências de barotrauma ${ }^{14,15}$.

\section{FREQÜÊNCIA RESPIRATÓRIA}

Recomendação: Utilizar freqüência respiratória entre 7 e 11 incursões por minuto.

\section{Grau de Recomendação: B}

Comentário: Na fase inicial da ventilação (modo controlado, paciente profundamente sedado), estudos mostram que freqüências maiores que 11 incursões por minuto em pacientes em crise de asma aguda e em ventilação mecânica estão associadas à ocorrência de hiperinsuflação dinâmica e maior risco de barotrau$\mathrm{ma}^{12,14,15}$.

\section{FLUXO INSPIRATÓRIO}

Recomendação: Utilizar fluxos inspiratórios elevados - acima de 60 L/min - se estiver ventilando em modo 
volume controlado (os modos limitados à pressão caracterizam-se por fluxos inspiratórios livres que atingem essa magnitude). Deve-se estar atento para os limites de pressão nas vias aéreas.

\section{Grau de Recomendação: B}

Comentário: No modo volume controlado, o uso de fluxos elevados permite um tempo inspiratório mais curto, maximizando o tempo expiratório (tempo expiratório entre 4 e $5 \mathrm{seg})^{11}$. Note que o aumento do fluxo inspiratório causa aumento da pressão de pico nas vias aéreas, em função do aumento da pressão resistiva. Porém, a pressão de pico parece não ser fator de risco para barotrauma, pois ela não se transmite diretamente aos alvéolos, ao contrário da pressão de platô que deve representar a pressão alcançada nos alvéolos ao final de inspiração.

É necessário observar que, quando o volume-minuto for baixo, o aumento do tempo expiratório obtido pela elevação do fluxo inspiratório não causa desinsuflação pulmonar significativa ${ }^{14-16}$.

\section{FRAÇÃO INSPIRADA DE OXIGÊNIO $\left(\mathrm{FIO}_{2}\right)$}

Recomendação: $A$ fração inspirada de oxigênio deve ser ajustada com base na gasometria arterial ou na oximetria de pulso, devendo-se usar a menor $\mathrm{FIO}_{2}$ que mantenha a $\mathrm{SaO}_{2}$ acima de $95 \%$.

\section{Grau de Recomendação: D}

Comentário: Habitualmente pacientes com asma não têm dificuldade de oxigenação e baixas $\mathrm{FIO}_{2}$ são suficientes. Se ocorrer hipoxemia, verificar presença de atelectasias, pneumotórax e/ou pneumonias concomitantes ou ainda presença de shunt intracardíaco direito-esquerda ${ }^{3}$. É importante também medir a autoPEEP, de modo a excluir a hiperinsuflação dinâmica como causa de hipoxemia.

\section{PRESSÃO POSITIVA AO FINAL DA EXPIRAÇÃO (PEEP)}

Recomendação: A PEEP poderá ser utilizada em casos seletos de crise de asma aguda, como uma tentativa de desinsuflação pulmonar. Deve ser utilizado em unidades especializadas e com monitoração adequada.

Grau de Recomendação: C

Comentário: A aplicação externa de PEEP pode, por ação mecânica, manter maior calibre nas vias aéreas e, assim, reduzir a resistência ao fluxo de ar. Entretanto, pelas características não homogêneas dos pulmões, algumas áreas podem sofrer hiperinsuflação. Por isso, deve ser feita tentativa de aplicação de PEEP na crise de asma aguda com monitoração das curvas de fluxo, pressão e volume. Se, com o aumento da PEEP, ocorrer desinsuflação, ela poderá ser aplicada. Se, pelo contrário, ocorrer hiperinsuflação, deverá ser evitado. Caso se opte pela tentativa de aplicação de PEEP externa, orienta-se a ventilação com modalidades limitadas à pressão, fixando-se o diferencial de pressão utilizado. Assim, à medida que se aumenta o valor da PEEP, monitora-se o volume corrente exalado. Se este se reduzir, é sinal de que está havendo piora na hiperinsuflação e a PEEP deverá ser reduzida. Se, por outro lado, o volume exalado aumentar, a PEEP está ocasionando desinsuflação pulmonar pode ser mantida ${ }^{17}$.

\section{HIPERCAPNIA PERMISSIVA}

Recomendação: A elevação da $\mathrm{PaCO}_{2}$ para valores acima do normal (até $90 \mathrm{mmHg}$ ), com pH acima de 7 , pode ser tolerada durante a ventilação mecânica na asma, caso seja necessária para diminuir a hiperinsuflação pulmonar.

Grau de Recomendação: B

Comentário: Como já descrito, a hiperinsuflação pulmonar é deletéria na ventilação mecânica do paciente asmático e as estratégias capazes de minimizar esse problema podem levar à hipoventilação do paciente, com conseqüente retenção de gás carbônico. Geralmente, a hipercapnia é bem tolerada e, pelos benefícios de se controlar a hiperinsuflação, deve ser aceita. Em situações de acidose grave, pode-se neutralizar o $\mathrm{pH}$ com o uso de bicarbonato em infusão lenta ${ }^{3,18-20}$.

\section{MONITORAÇÃO DA MECÂNICA PULMONAR}

Recomendação: A monitoração da mecânica respiratória e da hiperinsuflação pulmonar é aconselhável na crise asmática. Os principais parâmetros a se avaliar são: pressão de platô, pressão de pico, auto-PEEP e volume pulmonar ao final da inspiração. A medida seriada da resistência das vias aéreas é útil para avaliar a resposta ao tratamento broncodilatador e antiinflamatórios ${ }^{9,10,21 .}$.

Grau de Recomendação: B

A seguir estão comentários e graus de recomendação específicos para a monitorização de cada um desses parâmetros:

\section{PRESSÃO DE PLATÔ}

Comentário: Apresenta melhor correlação com a hiperinsuflação, pois como não há comprometimento 
importante da complacência do sistema respiratório na asma, sua elevação decorre do aprisionamento de ar nos pulmões, dando uma estimativa do auto-PEEP presente nas diversas unidades alveolares heterogeneamente acometidas. Recomenda-se que ela seja mantida no menor valor possível, lembrando-se dos limites de $35 \mathrm{cmH}_{2} \mathrm{O}$ para diminuição de risco de barotrau$\mathrm{ma}^{10,14,15}$

\section{Grau de Recomendação: D}

\section{PRESSÃO DE PICO}

Comentário: A medida isolada da pressão de pico não traz informações adequadas sobre a presença e a magnitude da hiperinsuflação, pois seu valor sofre grande influência da relação entre a resistência das vias aéreas (incluindo a cânula traqueal) e o fluxo inspiratório. Assim, por exemplo, elevações do fluxo podem reduzir a hiperinsuflação e, ao mesmo tempo, elevar a pressão de pico. Apesar das limitações, em geral, orienta-se mantê-la abaixo de $50 \mathrm{cmH}_{2} \mathrm{O}^{10,14,15}$.

Grau de Recomendação:

\section{AUTO-PEEP}

Comentário: A medida da auto-PEEP também traz informações sobre a hiperinsuflação. A forma mais prática de se medir a auto-PEEP é a estática, através da manobra de oclusão da válvula de exalação ao final da expiração, retardando-se o início do próximo ciclo respiratório. Essa medida exige que o paciente não execute esforço respiratório e expressa a média das pressões alveolares ao final da expiração de diferentes unidades em contato com as vias aéreas proximais (auto-PEEP estático). A auto-PEEP deve ser mantida abaixo de $15 \mathrm{cmH}_{2} \mathrm{O}$ para se diminuir a chance de ocorrência de barotrauma ${ }^{10,14,15}$.

\section{Grau de Recomendação: D}

\section{VOLUME PULMONAR AO FINAL DA INSPIRAÇÃO}

Comentário: É a técnica mais fidedigna de avaliação da hiperinsuflação pulmonar, mas seu uso é limitado pelas dificuldades de execução. Consiste na medida do volume de ar exalado durante longo período de apnéia, até que nenhum fluxo expiratório seja detectado. Este compreende o volumecorrente ofertado no ciclo anterior mais o volume adicional resultante da hiperinsuflação. Já se demonstrou que quando esse volume foi inferior a 1,4 L não se observaram complicações decorrentes de hiperinsuflação ${ }^{10,14,15}$.

Grau de Recomendação: C
OUTROS ASPECTOS RELACIONADOS AO CUIDADO DO PACIENTE COM CRISE ASMÁTICA AGUDA EM VENTILAÇÃO MECÂNICA

\section{ANALGÉSICOS E SEDATIVOS}

Recomendação: As medicações que promovem liberação de histamina, como morfina e meperidina devem ser evitadas.

\section{Grau de Recomendação: B}

Comentário: Resultados de estudos terapêuticos mostraram que morfina e meperidina podem liberar histamina, piorando as crises dos pacientes asmáticos, razão pela qual devem ser evitadas ${ }^{5,19,22,23}$.

\section{BLOQUEADORES NEUROMUSCULARES}

Recomendação: Os bloqueadores neuromusculares devem ser evitados ou, se absolutamente necessários, devem ser utilizados pelo menor tempo possível.

\section{Grau de Recomendação: B}

Comentário: Nas fases iniciais da ventilação mecânica na asma, para se alcançar o objetivo de minimizar a hiperinsuflação pulmonar, é fundamental o controle do volume minuto, o qual, muitas vezes, deverá ser mantido abaixo da demanda ventilatória do paciente. Eventualmente, pode ser necessário a suplementação da sedação com bloqueio neuromuscular. Como os bloqueadores neuromusculares podem levar à miopatia, sobretudo em pacientes que estão usando corticosteróide, eles devem ser aplicados pelo menor tempo possível (a duração do bloqueio neuromuscular parece relacionar-se com a ocorrência da miopatia) ${ }^{24}$.

\section{TRATAMENTOS VENTILATÓRIOS ALTERNATIVOS NA CRISE ASMÁTICA}

\section{AGENTES ANESTÉSICOS}

Recomendação: Os anestésicos inalatórios, como halotano, enflurano e isoflurano têm propriedades broncodilatadoras e podem ser considerados como tratamento de resgate em crise asmática refratária. No entanto, dados sobre a segurança e a eficácia desses agentes em pacientes asmáticos são escassos ${ }^{25}$.

Grau de Recomendação: C

\section{CIRCULAÇÃO EXTRACORPÓREA}

Recomendação: Em relatos anedóticos, o suporte de vida com circulação extracorpórea nas crises de asma aguda refratária tem sido utilizado com o intuito de manutenção da vida e da ventilação e oxigenação enquanto os pacientes se recuperam da crise aguda ${ }^{26-30}$. 
Grau de Recomendação: C

\section{MISTURA HÉLIO-OXIGÊNIO (HELIOX)}

Recomendações: A mistura hélio-oxigênio (Heliox) reduz a pressão resistiva na via aérea, por redução da turbulência do fluxo de $a^{31}$. O uso dessa mistura em pacientes com crise asmática em ventilação mecânica foi estudado em pequeno número de pacientes ${ }^{32-35}$. Pode ser usada como opção terapêutica em casos graves, porém com o cuidado de se aplicarem as fórmulas de correção para se obter $\mathrm{FIO}_{2}$ e volume-corrente adequado ${ }^{36}$, pois esses diferem dos valores ajustados no respirador quando o heliox é utilizado ${ }^{36,37}$.

\section{REFERÊNCIAS}

01. Gupta D, Keogh B, Chung KF et al - Characteristics and outcome for admissions to adult, general critical care units with acute severe asthma: a secondary analysis of the ICNARC Case Mix Programme Database. Crit Care, 2004;8:R112-R121.

02. McCoy L, Redelings M, Sorvillo F et al - A multiple cause-of-death analysis of asthma mortality in the United States, 1990-2001. J Asthma, 2005;42:757-763

03. McFadden ER Jr - Acute severe asthma. Am J Respir Crit Care Med, 2003;168:740-759.

04. Pierson DJ - Indications for mechanical ventilation in adults with acute respiratory failure. Respir Care, 2002;47:249-265.

05. Winters AC - Management of acute severe asthma. Crit Care Nurs Clin North Am, 2004;16:285-291.

06. Marini JJ - Partitioning the work-sparing effects of partial ventilatory support in airflow obstruction. Crit Care, 2004;8:101-102.

07. Darioli R, Perret C - Mechanical controlled hypoventilation in status asthmaticus. Am Rev Respir Dis, 1984;129:385-387.

08. Stather DR, Stewart TE - Clinical review: Mechanical ventilation in severe asthma. Crit Care, 2005;9:581-587.

09. Calverley PM, Koulouris NG - Flow limitation and dynamic hyperinflation: key concepts in modern respiratory physiology. Eur Respir J, 2005;25:186-199.

10. Blanch L, Bernabe F, Lucangelo $U$ - Measurement of air trapping, intrinsic positive end-expiratory pressure, and dynamic hyperinflation in mechanically ventilated patients. Respir Care, 2005;50:110-124.

11. Oddo M, Feihl F, Schaller MD et al - Management of mechanical ventilation in acute severe asthma: practical aspects. Intensive Care Med, 2006;32:501-510.

12. Leatherman JW, McArthur C, Shapiro RS - Effect of prolongation of expiratory time on dynamic hyperinflation in mechanically ventilated patients with severe asthma. Crit Care Med, 2004;32:1542-1545.

13. Adams BK, Cydulka RK - Asthma evaluation and management. Emerg Med Clin North Am, 2003;21:315-330.

14. Tuxen DV, Lane S - The effects of ventilatory pattern on hyperinflation, airway pressures, and circulation in mechanical ventilation of patients with severe air-flow obstruction. Am Rev Respir Dis, 1987;136:872-879.
15. Tuxen DV, Williams TJ, Scheinkestel CD et al - Use of a measurement of pulmonary hyperinflation to control the level of mechanical ventilation in patients with acute severe asthma. Am Rev Respir Dis, 1992;146:(5 Pt 1):1136-1142.

16. McCarthy DS, Sigurdson M - Lung elastic recoil and reduced airflow in clinically stable asthma. Thorax, 1980;35:298-302.

17. Caramez MP, Borges JB, Tucci MR et al - Paradoxical responses to positive end-expiratory pressure in patients with airway obstruction during controlled ventilation. Crit Care Med, 2005;33:1519-1528.

18. Shapiro JM - Management of respiratory failure in status asthmaticus. Am J Respir Med, 2002;1:409-416.

19. Han P, Cole RP - Evolving differences in the presentation of severe asthma requiring intensive care unit admission. Respiration, 2004;71:458462.

20. Tuxen DV - Permissive hypercapnic ventilation. Am J Respir Crit Care Med, 1994;150:870-874.

21. Dhand R - Ventilator graphics and respiratory mechanics in the patient with obstructive lung disease. Respir Care, 2005;50:246-261.

22. Graziani E, Petroianni A, Terzano C - Brittle asthma. Eur Rev Med Pharmacol Sci, 2004;8:135-138

23. Pagani JL, Oddo M, Schaller MD - Severe acute asthma. Rev Med Suisse Romande 2004;124:333-336.

24. De Jonghe $B$, Sharshar T, Lefaucheur JP et al - Paresis acquired in the intensive care unit: a prospective multicenter study. JAMA, 2002;288:28592867.

25. Saulnier FF, Durocher AV, Deturck RA et al - Respiratory and hemodynamic effects of halothane in status asthmaticus. Intensive Care Med, 1990;16:104-107.

26. Kukita I, Okamoto K, Sato T et al - Emergency extracorporeal life support for patients with near-fatal status asthmaticus. Am J Emerg Med, 1997;15:566-569.

27. Sakai M, Ohteki H, Doi K et al - Clinical use of extracorporeal lung assist for a patient in status asthmaticus. Ann Thorac Surg, 1996;62:885-887.

28. Tajimi K, Kasai T, Nakatani T et al - Extracorporeal lung assist for patient with hypercapnia due to status asthmaticus. Intensive Care Med 1988;14:588-589.

29. Leiba A, Bar-Yosef S, Bar-Dayan $Y$ et al - Early administration of extracorporeal life support for near fatal asthma. Isr Med Assoc J, 2003;5:600602.

30. Mabuchi N, Takasu H, Ito S et al - Successful extracorporeal lung assist (ECLA) for a patient with severe asthma and cardiac arrest. Clin Intensive Care, 1991;2:292-294.

31. Jolliet P, Tassaux D - Helium-oxygen ventilation. Respir Care Clin N Am, 2002;8:295-307.

32. Gluck EH, Onorato DJ, Castriotta R - Helium-oxygen mixtures in intubated patients with status asthmaticus and respiratory acidosis. Chest 1990;98:693-698.

33. Schaeffer EM, Pohlman A, Morgan S et al - Oxygenation in status asthmaticus improves during ventilation with helium-oxygen. Crit Care Med, 1999;27:2666-2670.

34. Kass JE, Castriotta RJ - Heliox therapy in acute severe asthma. Chest, 1995; $107: 757-760$

35. Ho AM, Lee A, Karmakar MK et al - Heliox vs air-oxygen mixtures for the treatment of patients with acute asthma: a systematic overview. Chest, 2003;123:882-890.

36. Tassaux D, Jolliet $P$, Thouret JM et al - Calibration of seven ICU ventilators for mechanical ventilation with helium-oxygen mixtures. Am J Respir Crit Care Med, 1999;160:22-32.

37. Burns SM - Ventilating patients with acute severe asthma: what do we really know? AACN Adv Crit Care, 2006;17:186-193. 